\title{
Alternative experimental evidence for chiral restoration in excited baryons
}

\author{
L. Ya. Glozman \\ Institute for Physics, Theoretical Physics Branch, \\ University of Graz, Universitätsplatz 5, A-8010 Graz, Austria
}

\begin{abstract}
Given existing empirical spectral patterns of excited hadrons it has been suggested that chiral symmetry is approximately restored in excited hadrons at zero temperature/density (effective symmetry restoration). If correct, this implies that mass generation mechanisms and physics in excited hadrons is very different as compared to the lowest states. One needs an alternative and independent experimental information to confirm this conjecture. Using very general chiral symmetry arguments it is shown that strict chiral restoration in a given excited nucleon forbids its decay into the $N \pi$ channel. Hence those excited nucleons which are assumed from the spectroscopic patterns to be in approximate chiral multiplets must only "weakly" decay into the $N \pi$ channel, $\left(f_{N^{*} N \pi} / f_{N N \pi}\right)^{2} \ll 1$. However, those baryons which have no chiral partner must decay strongly with a decay constant comparable with $f_{N N \pi}$. Decay constants can be extracted from the existing decay widths and branching ratios. It turnes out that for all those well established excited nucleons which can be classified into chiral doublets $N_{+}(1440)-N_{-}(1535), N_{+}(1710)-N_{-}(1650), N_{+}(1720)-N_{-}(1700)$, $N_{+}(1680)-N_{-}(1675), N_{+}(2220)-N_{-}(2250), N_{+}(?)-N_{-}(2190), N_{+}(?)-N_{-}(2600)$, the ratio is $\left(f_{N^{*} N \pi} / f_{N N \pi}\right)^{2} \sim 0.1$ or much smaller for the high-spin states. In contrast, the only well established excited nucleon for which the chiral partner cannot be identified from the spectroscopic data, $N(1520)$, has a decay constant into the $N \pi$ channel that is comparable with $f_{N N \pi}$. This gives an independent experimental verification of the chiral symmetry restoration scenario.

PACS numbers: 11.30.Rd, 14.20.Gk, 12.38.Aw
\end{abstract}

1. Introduction. The question of mass generation in QCD and the related question about interconnections of chiral symmetry and confinement are central for QCD in the infrared. To answer these questions one must understand spectral and other properties of hadrons in the light quark sector. A long history of studies in this area has not suggested any satisfactory answer. Given existing spectroscopic patterns it has recently been suggested that chiral and $U(1)_{A}$ symmetries of the QCD Lagrangian, strongly broken in the vacuum, get approximately restored in excited hadrons [1, 2, 3, 4] , for a review see ref. [5]. This would imply that physics and in particular mass generation mechanisms in the lowest and excited hadrons are very different.

The nucleon excitation spectrum is shown in Fig. 1 of ref. [5]. Only well-established states have to be seriously considered. It is well seen that there is no obvious chiral partner to the nucleon. This implies that chiral symmetry breaking effects are very strong in the given case and consequently the chiral symmetry is realized nonlinearly in the nucleon [7]. This is consistent with the well-known result that the mass of the nucleon is (at least mostly) induced by the quark condensate of the vacuum [8]. This is supported by the empirical success of the Goldberger-Treiman relation, which also implies that the nucleon mass comes from the chiral symmetry breaking in the vacuum [9]. Another clear indication of the strong chiral symmetry breaking effects in the nucleon is a large pion-nucleon coupling constant. All empirical successes of chiral perturbation theory for the nucleon [10] also implicitly rely on the assumption that the nucleon mass is due to chiral symmetry breaking in the vacuum. These results, taken together, suggest that there is no chiral partner to the nucleon.

Obvious approximate parity doublets are observed in the $1.7 \mathrm{GeV}$ region. These have been assigned to the $(0,1 / 2)+$ $(1 / 2,0)$ representation of the parity-chiral group because there are no approximately degenerate doublets in the same mass region in the spectrum of the delta-resonance [3,5]. A clear testable prediction of the chiral symmetry restoration scenario is an existence of chiral partners of the well established high-lying resonances $N(2190)$ and $N(2600)$. A dedicated experimental search of these missing states can be undertaken [11]. A similar situation takes place in the Delta-spectrum.

While these parity doubling patterns are impressive, they are still only suggestive, because so far no other complementary experimental data would independently tell us that these parity doublets are due to effective chiral symmetry restoration. Strict chiral restoration in a given baryon would imply that its diagonal axial charge is zero and hence the diagonal coupling constant to the pion must vanish [5, 12, 13, 14]. An experimental verification of the smallness of these quantities for approximate parity doublets would be a confirmation of the chiral restoration scenario. However, it is unclear how to extract this information from the existing data. Hence it is important to find alternative experimental signatures of chiral restoration.

A different assignement of excited baryons was discussed in ref. 15], where some of the excited nucleons below the $1.7 \mathrm{GeV}$ region have been combined with the delta-resonances into quartets of the $(1,1 / 2)+(1 / 2,1)$ representation. 
Such an assignement requires a large pion-baryon coupling constant [15], because there is a large mass splitting between the assumed members of the quartets, and hence does not correspond to the effective chiral symmetry restoration regime.

There is rich experimental data on strong decays of excited hadrons. It turnes out that the effective chiral restoration implies a very strong selection rule. Namely, it predicts that if chiral symmetry is completely restored in a given excited nucleon $(B)$, then it cannot decay into the $\pi N$ channel, i.e. the coupling constant $f_{B N \pi}$ must vanish. This selection rule is based exclusively on general properties of chiral symmetry, what will be demonstrated below, and hence is modelindependent. Such a coupling can be extracted from the existing decay data [16, 17]. Then an independent verification of the chiral symmetry restoration scenario would require that those excited nucleons that are approximate parity doublets, only "weakly" decay into the $\pi N$ channel, $\left(f_{B N \pi} / f_{N N \pi}\right)^{2} \ll 1$, and, on the contrary, those excited nucleons, to which the chiral partner cannot be identified from the spectroscopic data, must decay strongly, $\left(f_{B N \pi} / f_{N N \pi}\right)^{2} \sim 1$.

2. Let us review first the question whether there are or not chiral symmetry constraints for a magnitude of the $\pi N N$ coupling (well known from the sigma-model derivations [6, 7, 9]). As a starting point for the chiral symmetry analysis it is always useful to consider the linear realization of chiral symmetry. The standard assumption is that the pion field (together with the sigma field or quark condensate) transform as $(1 / 2,1 / 2)$ of $S U(2)_{L} \times S U(2)_{R}$. Throughout the paper we ignore small quark masses. The very absence of an independent chiral partner to the nucleon implies that the nucleon field $N$ in the Wigner-Weyl mode transforms as $(0,1 / 2)+(1 / 2,0)$ and is massless. Its chiral partner is $\gamma_{5} N$. The axial transformation law is then

$$
N \rightarrow \exp \left(\imath \gamma_{5} \frac{\theta_{A}^{a} \tau^{a}}{2}\right) N
$$

Then one can construct the $\pi N N$ vertex as

$$
\sim \bar{N} e^{i \gamma_{5} \frac{\vec{\pi} \cdot \vec{\tau}}{f_{\pi}}} N
$$

This vertex is chiral-invariant (chiral scalar) because the axial rotation of the nucleon field is compensated by the rotation of the pion field. A strength of the interaction is fixed by the coupling constant which is an external parameter and can have any arbitrary value. Hence we conclude that chiral symmetry does not restrict the $\pi N N$ interaction which can be arbitrarily strong. In the Nambu-Goldstone mode the nucleon field becomes massive due to its coupling with the chiral order parameter. The axial current conservation connects this nucleon mass and axial charge via the Goldberger-Treiman relation with the pion-nucleon coupling constant, $g_{N N \pi}=\frac{g_{A} M_{N}}{f_{\pi}}$. Hence the large pion-nucleon coupling constant encodes a physical origin of the nucleon mass as due to chiral symmetry breaking in the vacuum. In other words, the large $\pi N N$ coupling constant can be used as a natural unit for strong chiral symmetry breaking effects in a baryon.

3. Assume that we have a free $I=1 / 2$ chiral doublet $B$ in the $(0,1 / 2)+(1 / 2,0)$ representation and there are no chiral symmetry breaking terms. This doublet is a column [9]

$$
B=\left(\begin{array}{c}
B_{+} \\
B_{-}
\end{array}\right)
$$

where the bispinors $B_{+}$and $B_{-}$have positive and negative parity, respectively, because the parity on the doublet space is defined to be

$$
P: \quad B(\vec{x}, t)=\sigma_{3} \gamma_{0} B(-\vec{x}, t) .
$$

The chiral transformation law under the $(0,1 / 2) \oplus(1 / 2,0)$ representation provides a mixing of two fields $B_{+}$and $B_{-}$ [21]

$$
B \rightarrow \exp \left(\imath \frac{\theta_{V}^{a} \tau^{a}}{2}\right) B ; \quad B \rightarrow \exp \left(\imath \frac{\theta_{A}^{a} \tau^{a}}{2} \sigma_{1}\right) B
$$

Here $\sigma_{i}$ is a Pauli matrix that acts in the $2 \times 2$ space of the parity doublet. Then the chiral-invariant Lagrangian of the free parity doublet is given as 


$$
\begin{aligned}
\mathcal{L}_{0} & =i \bar{B} \gamma^{\mu} \partial_{\mu} B-m_{0} \bar{B} B \\
& =i \bar{B}_{+} \gamma^{\mu} \partial_{\mu} B_{+}+i \bar{B}_{-} \gamma^{\mu} \partial_{\mu} B_{-}-m_{0} \bar{B}_{+} B_{+}-m_{0} \bar{B}_{-} B_{-} .
\end{aligned}
$$

Alternative forms for this Lagrangian can be found in refs. [18, 19].

A crucial property of this Lagrangian is that the fermions $B_{+}$and $B_{-}$are exactly degenerate and have a nonzero chiral-invariant mass $m_{0}$. In contrast, for usual (Dirac) fermions chiral symmetry in the Wigner-Weyl mode restricts particles to be massless.

From the axial transformation law (5) one can read off the axial charge matrix, which is $\gamma_{5} \sigma_{1}$. Hence the diagonal axial charges of the opposite parity baryons are exactly $0, g_{+}^{A}=g_{-}^{A}=0$, while the off-diagonal axial charge is 1 , $\left|g_{+-}^{A}\right|=\left|g_{-+}^{A}\right|=1$. This is another crucial property that distinguishes the parity doublets from the Dirac fermions where $g^{A}=1$. The axial vector current conservation translates this axial charge matrix into the $\pi B_{ \pm} B_{ \pm}$coupling constants which are zero. Hence a small (vanishing) value of the pion-baryon coupling constant taken together with the large baryon mass would tell us that the origin of this mass is not due to chiral symmetry breaking in the vacuum. As discussed in the introduction part an experimental verification of the smallness of the diagonal axial charges or smallness of the pion-baryon coupling constants would be a direct verification of the chiral symmetry restoration scenario in excited nucleons. It is unclear, however, how to measure these quantities.

4. Consider a possible $B_{ \pm} N \pi$ vertex. Since these parity doublet baryons $B_{+}, B_{-}$are in the $(0,1 / 2) \oplus(1 / 2,0)$ representation, like the nucleon, on the first value the chiral-invariant vertex $B N \pi$ is possible. This is incorrect, however. The reason is that the axial transformation laws for the nucleon field and for the parity doublet fields are very different, because they live in different linear spaces. While the axial rotation of the nucleon field is compensated by the chiral transformation of the pion field in the vertex (2), so this vertex is chiral-invariant, such a compensation is impossible if one of the legs of this vertex is substituted by $B_{ \pm}$. Consequently one cannot construct a chiral-invariant interaction vertex $B_{ \pm} N \pi$. Hence decay of the parity doublets with completely restored chiral symmetry into $N \pi$ is impossible. 222] However, their decay into e.g. $N \rho$ or $N \pi \pi$ is not forbidden.

If, in contrast, the excited baryon has no chiral partner, then its mass, like in the nucleon case is exclusively due to chiral symmetry breaking in the vacuum. Its axial charge should be comparable with the nucleon axial charge. Then nothing forbids its strong decay into $N \pi$. One then expects that the decay coupling constant should be of the same order as the pion-nucleon coupling constant. These two extreme cases suggest that a magnitude of the $B N \pi$ decay constant can be used as an indicator of the mass origin.

5. In reality, of course, chiral symmetry is never completely restored in excited hadrons. To account a small amount of chiral symmetry breaking contribution one must add into Lagrangian terms that provide a coupling of the given hadron with the vacuum. Then the baryon mass can be split into two different parts: (i) chiral-invariant $m_{0}$ and (ii) chiral "non-invariant" $m^{\prime}$. The latter is due to coupling of the hadron with the chiral-noninvariant vacuum (i.e. with the quark condensate). However, if the coupling with the quark condensate is weak and consequently the chiral symmetry breaking mass $m^{\prime}$ is small, $m^{\prime} \ll m_{0}$, then the decay into $N \pi$ must be strongly suppressed. The magnitude of $m^{\prime}$ can be determined from the chiral asymmetry parameter [4, 5]

$$
\chi=\frac{\left|M_{B_{+}}-M_{B_{-}}\right|}{M_{B_{+}}+M_{B_{-}}} .
$$

This papameter can be interpreted as a part of the hadron mass due to chiral symmetry breaking in the vacuum. Chiral asymmetries of the excited nucleons in the $1.7 \mathrm{GeV}$ mass region and above are typically within 0.02 . Hence, if these parity doublets are indeed due to the effective symmetry restoration, then their decay into $N \pi$ must be very strongly suppressed.

6. Now we can formulate predictions of the chiral symmetry restoration scenario. If a state is a member of an approximate chiral multiplet and $\chi \ll 1$, then its decay into $N \pi$ must be strongly suppressed, $\left(f_{B N \pi} / f_{N N \pi}\right)^{2} \ll 1$. If, on the contrary, this excited hadron has no chiral partner and hence its mass is due to chiral symmetry breaking in the vacuum, then it should strongly decay into $N \pi$ and hence $\left(f_{B N \pi} / f_{N N \pi}\right)^{2} \sim 1$. In the following section we demonstrate that this prediction is in accord with the existing data.

7. Given the well-known Rarita-Schwinger formalism for the higher-spin fields, one can construct phenomenological $B N \pi$ Lagrangians. Then decay constants $f_{B N \pi}$ can be extracted from the $B \rightarrow N+\pi$ decay widths, see e.g. [16, 17]. The pion-nucleon coupling constant is well-known, $f_{N N \pi}=1$.0. In Table 1 we show ratios $\left(f_{B N \pi} / f_{N N \pi}\right)^{2}$ for all 
TABLE I: Chiral multiplets of excited nucleons. Comments: (i) All these states are well established and can be found in the Baryon Summary Table of the Review of Particle Physics. (ii) There are two possibilities to assign the chiral representation: $(1 / 2,0) \oplus(0,1 / 2)$ or $(1 / 2,1) \oplus(1,1 / 2)$ because there is a possible chiral pair in the $\Delta$ spectrum with the same spin with similar mass. (iii) The missing chiral partner is predicted.

\begin{tabular}{|llllll|}
\hline Spin & Chiral multiplet & Representation & $\chi$ & $\left(f_{B_{+} N \pi} / f_{N N \pi}\right)^{2}-\left(f_{B_{-} N \pi} / f_{N N \pi}\right)^{2}$ & Comment \\
\hline $1 / 2$ & $N_{+}(1440)-N_{-}(1535)$ & $(1 / 2,0) \oplus(0,1 / 2)$ & 0.032 & $0.15-0.026$ & $(\mathrm{i})$ \\
$1 / 2$ & $N_{+}(1710)-N_{-}(1650)$ & $(1 / 2,0) \oplus(0,1 / 2)$ & 0.02 & $0.0030-0.026$ & $(\mathrm{i})$ \\
$3 / 2$ & $N_{+}(1720)-N_{-}(1700)$ & $(1 / 2,0) \oplus(0,1 / 2)$ & 0.01 & $0.023-0.13$ & $(\mathrm{i})$ \\
$5 / 2$ & $N_{+}(1680)-N_{-}(1675)$ & $(1 / 2,0) \oplus(0,1 / 2)$ & 0.002 & $0.18-0.012$ & $(\mathrm{i})$ \\
$7 / 2$ & $N_{+}(?)-N_{-}(2190)$ & see comment (ii) $?$ & $?-0.00053$ & $(\mathrm{i}),(\mathrm{ii}),(\mathrm{iii})$ \\
$9 / 2$ & $N_{+}(2220)-N_{-}(2250)$ & see comment (ii) & 0.01 & $0.000022-0.0000020$ & $(\mathrm{i}),(\mathrm{ii})$ \\
$11 / 2$ & $N_{+}(?)-N_{-}(2600)$ & see comment (ii) $?$ & $?-0.000000064$ & (i),(ii),(iii) \\
\hline \hline $3 / 2$ & $N_{-}(1520)$ & no chiral partner - & 2.5 & (i) \\
\hline
\end{tabular}

well-established states. It is well seen that this ratio is $\sim 0.1$ or smaller for approximate $J=1 / 2,3 / 2,5 / 2$ parity doublets. For the high-spin states this ratio is practically vanishing. This is consistent with the recent demonstration of the large J-rate of chiral restoration within the only known exactly solvable confining and chirally-symmetric model [20]. In contrast, the only well-known state $J=3 / 2, N_{-}(1520)$, where a chiral partner is missing in the spectrum, decays very strongly into the $N \pi$ channel. Hence chiral symmetry breaking effects are as large in this case as in the nucleon. Consequently its physical origin should be very different as compared to all other resonances which can be classified into parity doublets.

In conclusion, the effective chiral symmetry restoration conjecture is now supported by strong decays of excited nucleons. A decay of the strict parity doublets into the $N \pi$ channel is forbidden by chiral symmetry, assuming that there is no independent chiral partner to the nucleon. Hence a fraction $\Gamma_{i} / \Gamma$ of the $N \pi$ decay channel becomes small, even though decays into other channels (not protected by chiral symmetry) are strongly suppressed by the phase space factor.

L.Ya.G. is grateful to Tom Cohen for useful comments and to Dan Riska for correspondence. Support of the Austrian Science Fund through grant P19168-N16 is acknowledged.

[1] L. Ya. Glozman, Phys. Lett. B 475, 329 (2000).

[2] T. D. Cohen and L. Ya. Glozman, Phys. Rev. D 65, 016006 (2002).

[3] T. D. Cohen and L. Ya. Glozman, Int. J. Mod. Phys. A 17, 1327 (2002).

[4] L. Ya. Glozman, Phys. Lett. B 539, 257 (2002); Phys. Lett. B 541, 115 (2002); Phys. Lett. B 587, 69 (2004); Int. J. Mod. Phys. A 21, 475 (2006).

[5] L. Ya. Glozman, Phys. Rep. 444, 1 (2007).

[6] M. Gell-Mann, M. Levy, Nuovo Cimento 16, 705 (1960).

[7] S. Weinberg, Phys. Rev. 166, 1568 (1968).

[8] B. L. Ioffe, Nucl. Phys. B 188, 317 (1981); E: B 191, 591 (1981).

[9] B. W. Lee, Chiral Dynamics, Gordon and Breach, New York, 1972

[10] U-G. Meissner, Chiral QCD: Baryon Dynamics, in: At the frontiers of Particle Physics. Handbook of QCD., edited by M. Shifman, v. 1, p. 417, World Sc. 2001.

[11] A. Sibirtsev, J. Haidenbauer, S. Krewald, T.-S.H. Lee, U.-G- Meissner, A. Thomas, arXiv: 0706.0183 [nucl-th].

[12] L. Ya. Glozman, A. V. Nefediev, Phys. Rev. D 73, 074018 (2006).

[13] R. L. Jaffe, D. Pirjol, A. Scardicchio, Phys. Rev. D 74, 057901 (2006); Phys. Rev. Lett. 96, 121601 (2006).

[14] R. L. Jaffe, D. Pirjol, A. Scardicchio, Phys. Rep. 435, 157 (2006).

[15] D. Jido, T. Hatsuda, T. Kunihiro, Phys. Rev. Lett. 84, 3252 (2000).

[16] W. K. Cheng, C. W. Kim, Phys. Rev. 154, 1525 (1967).

[17] D. O. Riska and G. E. Brown, Nucl. Phys. A 679, 577 (2001).

[18] C. DeTar, T. Kunihiro, Phys. Rev. D 39, 2805 (1989).

[19] D. Jido, M. Oka, A. Hosaka, Progr. Theor. Phys. 106, 873 (2001).

[20] R. F. Wagenbrunn, L. Ya. Glozman, Phys. Lett. B 643, 98 (2006); Phys. Rev. D 75, 036007 (2007).

[21] Note that the axial transformation given in [9] is incorrect as it breaks chiral symmetry of the kinetic term. The correct axial transformation is given in ref. [5]. 
[22] This statement can also be proven in the following way. Assume that a $\pi N$ decay of an exact parity doublet is possible. Then there must be a self-energy contribution $B_{ \pm} \rightarrow \pi N \rightarrow B_{ \pm}$into its mass. Then the axial rotation (5) would require that the S-wave $\pi N$ state transforms into the $\mathrm{P}$-wave $\pi N$ state. However, in the Nambu-Goldstone mode the axial rotations of the pion and nucleon states are fixed - these are the nonlinear axial transformations [13, 14]. Given these well known axial transformation properties of the Goldstone boson and nucleon it is not possible to rotate the S-wave $\pi N$ state into the $\mathrm{P}$-wave $\pi N$ state. Therefore, there cannot be any $\pi N$ self-energy component in $B_{ \pm}$. Hence a decay $B_{ \pm} \rightarrow \pi N$ is forbidden. 